\title{
MONITOREO EN LÍNEA DE LA FRECUENCIA CARDÍACA POR IMAGEN FOTOPLETISMOGRAFÍA MEDIANTE FILTRADO POR KALMAN.
}

\author{
Juan-Carlos Cobos-Torres \\ Universidad Carlos III de Madrid-Leganés, 100294413@alumnos.uc3m.3s \\ Mohamed Abderrahim \\ Universidad Carlos III de Madrid-Leganés, mohamed@ing.uc3m.es
}

\begin{abstract}
Resumen
En este trabajo se presenta un método para la medición sin contacto de la frecuencia cardíaca mediante la técnica de imagen fotopletismografia, a través de la aplicación de un filtro basado en la técnica de Kalman. Este método es imperceptible para los seres humanos, se caracteriza por un coste computacional bajo y es robusto ante artefactos. El enfoque utiliza el análisis de la señal pletismográfica recogida desde una cámara de color estándar para estimar la frecuencia cardíaca. El sistema calcula el seguimiento de la señal obtenida, con el cual logra estimar y filtrar el desplazamiento de la señal promediada, eliminando de manera potente los artefactos. Además, cuenta con un sistema de apreciación de error que permite una estimación de la frecuencia cardiaca de manera eficiente. Finalmente, se obtuvo una buena estimación de los signos vitales de diez individuos. El análisis de Bland y Altman muestra una fuerte correlación entre la medición del sistema estándar y el procedimiento propuesto.
\end{abstract}

Palabras Clave: Fotopletismografía, Filtro de Kalman, Frecuencia Cardíaca, medición en línea.

\section{INTRODUCCIÓN}

La detección y búsqueda de variaciones pequeñas en el color de la piel producidas por la irrigación sanguínea, se han vuelto un parámetro determinante para la medición de signos vitales en pacientes, ya que cuenta con la ventaja sobre los métodos tradicionales de no tener contacto físico con el paciente. Estas variaciones que son imperceptibles para el ojo humano, pueden ser adquiridas por los dispositivos de captura de imágenes.

Dispositivos como la cámara de teléfonos, tabletas electrónicas o la cámara web de un ordenador son parte de nuestra vida diaria. Son una herramienta económica y simple para la captura de imágenes en línea, así como para el procesamiento de la señal plestimográfica mediante los cambios de coloración de la piel. Proceso que se denomina imagen fotopletismografía PPGI (photoplethysmographic image).

Para el procesamiento de las señales por PPGI existe diversidad de metodologías, todas con enfoques distintos en la etapa de filtrado. Así un primer método que funciona de manera robusta con iluminación natural y estable, se presenta en [1]. Utiliza un filtro pasa banda, Butterworth de $4^{\circ}$ orden, el cual le da una tolerancia baja a los artefactos e interferencias.

Hay tres parámetros que determinan estos denominados artefactos: distancia entre la cámara y la zona de la piel, la iluminación y factores propios de la piel como la pigmentación. Todos estos factores sumados forman una señal de muy baja frecuencia o una línea base que es variable en el tiempo. Con este tipo de señales los filtros convencionales no son apropiados, pues presentan diversos problemas como la necesidad de un gran número de coeficientes, problemas con la estabilidad y la banda de paso entre otros, en el caso de utilizar filtros pasa altos con frecuencias bajas.

Otra opción es lo contrario, utilizar un filtro pasa bajo, para estimar la componente de frecuencia baja, esta estimación se restará de la señal original. Se tendrá un número pequeño de coeficientes, pero con problemas en los tiempos de estabilización, por lo que movimientos rápidos, cambios de iluminación no se estimarán adecuadamente. Otro trabajo que utiliza el mismo tipo de filtrado es Eurelian Magnification [2]. Son capas de filtrado pasa banda de tipo Butterworth, con la diferencia que al conseguir la señal deseada, ésta es amplificada para que los cambios sean visibles, por ejemplo en este caso se visibilizan los cambios de color en la piel producidos por la perfusión sanguínea.

Otro tipo de filtrado son los de tipo estadístico, el análisis independiente de componentes ICA (Independent Component Analysis) es una técnica 
que se basa en la separación ciega de fuentes BSS (Blind Source Separation). Funciona bajo la condición que la señal a ser analizada es consecuencia de la mezcla desconocida de otras señales. Así [3, 4] presenta un método robusto y tolerable a movimientos suaves, y lo único que opaca está metodología es su coste computacional alto, por la fuerte base matemática y estadística del método.

De aquí, la búsqueda de otras alternativas como el análisis de componentes principales PCA (Principal Component Analysis) técnica también basada en la BSS. Existe una reducción de cálculo al descartar elementos del conjunto de datos con un nivel elevado de varianza, es decir los elementos de orden bajo son los que contendrán los aspectos relevantes de la señal como es el ritmo cardíaco. Este método se presenta en [5], obteniendo resultados similares al método ICA y con un tiempo menor de computo. De manera similar en [6] se presenta un método basado en esta metodología de filtrado, sin la presencia de un análisis en línea y mediante la colocación del dedo del paciente sobre la cámara del teléfono móvil, por lo cual se deduce que el coste computacional aún es elevado. Además, no se ha podido encontrar un estudio en el cual se valide su estabilidad y precisión en presencia de artefactos.

Otra metodología estadística basada en BSS es mediante el análisis de discriminante lineal LDA (Linear Discriminant Analysis), se presenta en [7], es un método relacionado con el PCA. La diferencia reside en que PCA, es una técnica de reducción de características que trata de trasladar un conjunto de vectores a una dimensión menor, minimizando el error a la hora de realizar la reducción de tamaño. En cambio, LDA se centra reducir las características necesarias para maximizar la distancia entre las clases, pero al final ambos métodos reducen la cantidad de datos. La metodología realiza mediciones en línea con un enfoque llamativo, cuenta con una etapa de pos filtrado, primero un filtro pasa banda (no especifican los autores el tipo), y luego mediante la máquina de estados finitos que busca una relación de la medida actual, con las lecturas anteriores.

Otra metodología usada es mediante la parametrización de características de piel, basándose en modelos de la piel. En el trabajo presentado en [8], se presenta un método que reproduce el funcionamiento del oxímetro de pulso, basándose en dos condiciones: la absorción de luz en la piel y la iluminación deberán ser constantes. El método modela la concentración de melanina (pigmento de la piel que según su proporción le da una tonalidad más obscura) y de la hemoglobina. La concentración de melanina es tratada como una señal línea base $u$ offset y la hemoglobina como la señal variable deseada. Esto está bien en teoría, pero en la realidad, las condiciones son variables, ya que variaciones en la radiación UV, iluminación en general, $\mathrm{u}$ movimientos van a cambiar el valor de este offset. Se observa que el coste computacional es bajo, aunque el análisis que se presenta no funciona en línea, sino mediante el análisis de videos. Además, es inexistente algún sistema de seguimientos de región de interés, por lo cual los autores afirman que movimientos considerables pueden producir mediciones inexactas.

Otro método utiliza el análisis del fondo de las imágenes, con lo cual busca corregir cambios de iluminación en la cara del paciente, se presenta en [9]. Consta de un filtro temporal de tres etapas: el seguimiento de señal para disminuir el offset, el cálculo de media móvil para eliminar segmentos de señal con una desviación elevada y finalmente con una ventana hamming para delimitar las frecuencias de interés. Junto a un buen seguimiento de la región de interés o rostro, lo convierten en un sistema bastante robusto. Pero con la desventaja que solo funciona en ambientes controlados sin fondo cambiante.

Por último, el trabajo detallado en [10], es un trabajo prolijo, en el cual se desarrolla: un modelo de la piel, un método para aislar los cambios en la iluminación $\mathrm{y}$ un conjunto de filtros temporales, para reducir el offset de la señal. Además, mediante un filtro de media móvil se mejora la reducción del offset. El análisis se realiza en dos zonas distintas del rostro, para encontrar la señal PPGI más fuerte, la cual se presenta como la medida buscada. El método de análisis utilizado es ICA, con lo cual a pesar de la estructura robusta del sistema tiene un coste computacional elevado (el análisis de 30 segundos de vídeo se demora aproximadamente 7 minutos, sin tomar en cuenta el tiempo de seguimiento).

Después de un análisis global de la adquisición, procesamiento y filtrado que son usados en la técnica de PPGI, en este trabajo, se propone un método de medida de la frecuencia cardíaca, robusto y con un coste computacional bajo, características fundamentales que le permite trabajar en línea. En resumen nuestras contribuciones son:

1. Mediante la aplicación del filtro de Kalman se genera un filtro de deducción de tendencia que permite eliminar artefactos y el offset de la señal pletismográfica.

2. Un sistema de apreciación de error que permite una estimación de la frecuencia cardíaca de manera robusta.

3. Se presenta un algoritmo simple con un coste computacional bajo que funciona en línea. 


\section{MATERIALES Y MÉTODOS}

El objetivo del presente trabajo es determinar la frecuencia cardíaca en línea mediante PPGI, para contar con un sistema robusto a artefactos y con un coste computacional bajo. Para el procesamiento de imágenes y selección de una pequeña región en la frente del paciente se utilizó como base el lenguaje de programación Python y diversas bibliotecas que se detallarán en la sección 2.3. Los vídeos son adquiridos en línea desde una cámara digital, la Figura 1 esquematiza el experimento. Con el fin de poder comprobar la frecuencia cardíaca medida se utiliza un pulsímetro de dedo, para conseguir valores de referencia obtenidos con los estándares de medición.

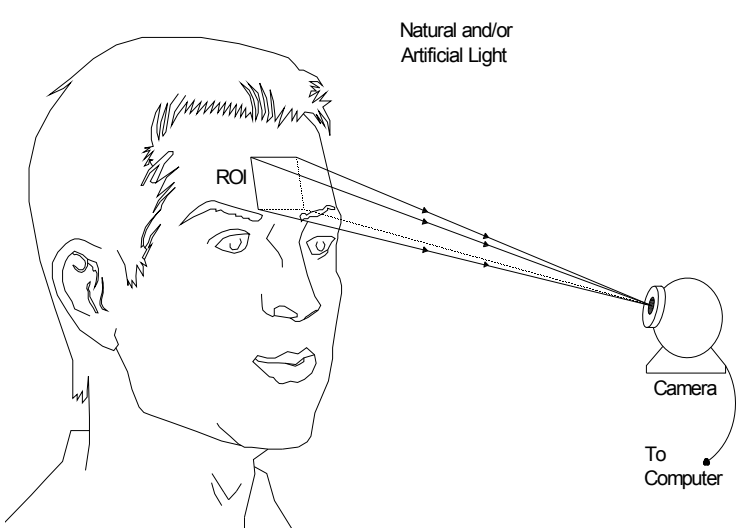

Figura 1: Configuración del experimento.

\subsection{POBLACIÓN}

Se trabajó con personas de distinta edad y sexo para estructurar una muestra heterogénea que permita demostrar la eficiencia del método propuesto, con participantes voluntarios que se pueden observar sus características en la tabla 1, todos sanos y sin complicaciones aparentes. Durante las mediciones estaban sentados frente a la cámara ubicada a una distancia de $50 \mathrm{~cm}$, aproximadamente.

Tabla 1: Detalle de población del experimento.

\begin{tabular}{|l|l|l|l|}
\hline & Edad & Sexo & \# Indiv. \\
\hline Niños & $<18$ & $\begin{array}{l}1 \mathrm{Fem}, \\
1 \mathrm{Mas} .\end{array}$ & 2 \\
& & $\begin{array}{l}3 \mathrm{Fem}, \\
3 \mathrm{Mas} .\end{array}$ & 6 \\
\hline Adultos & $>18 \mathrm{y}<50$ & $\begin{array}{l}1 \mathrm{Fem}, \\
1 \mathrm{Mas} .\end{array}$ & 2 \\
\hline Mayores & $>50$ & \\
\end{tabular}

\subsection{HARDWARE}

La cámara utilizada es una WebCam con un sensor CMOS OV9710 HD, con un formato del sensor de imagen de $1 / 4$ que proporciona WXGA (1280 x 800) y frecuencia máxima de captura de 30 fps. Permite la calibración directa de la exposición, control de ganancia, balance de blancos, entre otros. El sensor óptico utiliza un filtro de color con patrón Bayer y una matriz BG / GR dispuesto en un estilo de línea alterna.

\subsection{DESCRIPCIÓN DEL MÉTODO UTILIZADO PARA EL SISTEMA}

La estructura general utilizada para la obtención de la frecuencia cardíaca es detallada en la Figura 2.

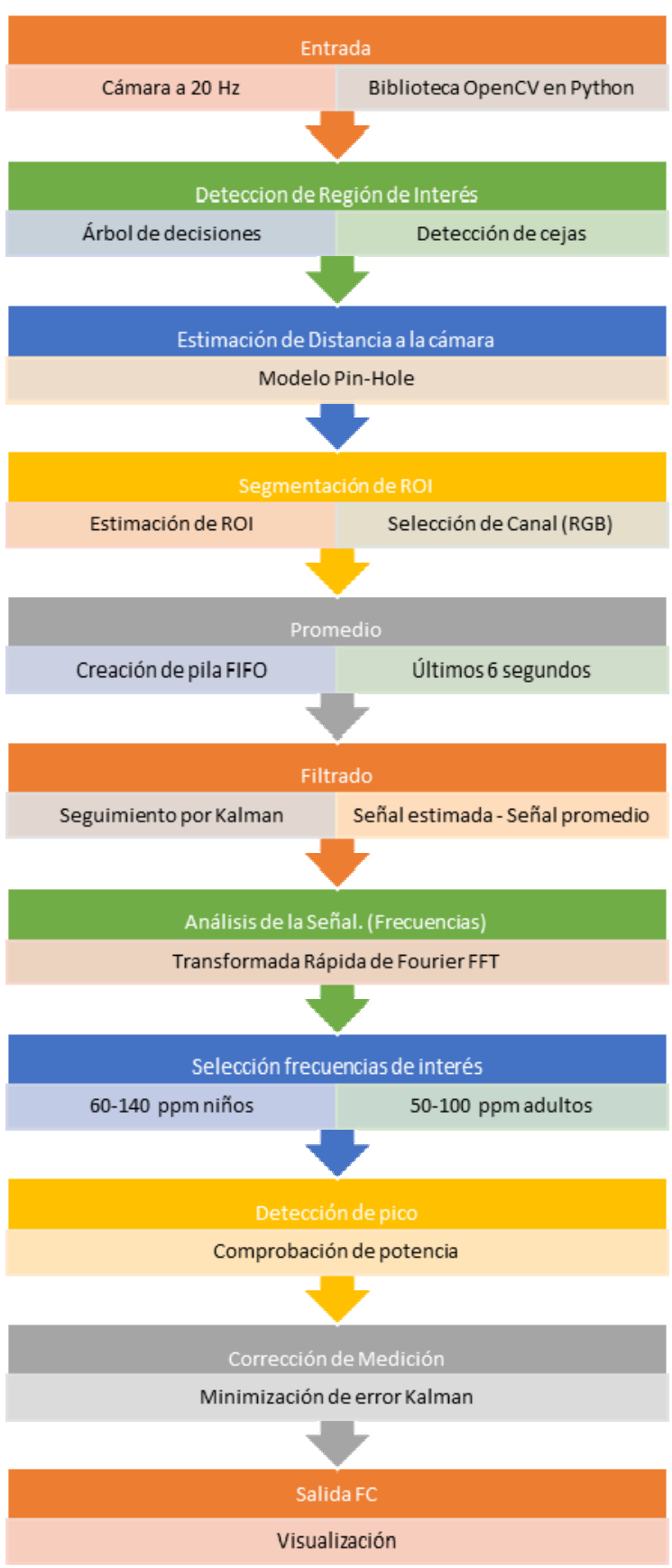

Figura 2: Secuencia utilizada para la medición de la frecuencia cardíaca por PPGI. 
El método consiste en la adquisición de imágenes mediante la cámara digital, este experimento se desarrolló bajo el lenguaje de programación Python y utilizando la biblioteca OpenCV. La captura de imágenes se realiza mediante la cámara digital a una frecuencia de $20 \mathrm{~Hz}$, con una resolución de 640x480 píxeles. Además, se realizan las siguientes calibraciones de la cámara: exposición para reducir posible efecto de desenfoque por movimiento, saturación para mejorar la sensibilidad de la cámara en cada uno de los sensores (RGB) del sensor CMOS y se calibra el balance de blancos. Independientemente del tipo de iluminación se busca un nivel de equilibrio entre los niveles de sensibilidad de la intensidad en los canales R y B.

Para la selección de la ROI, se utiliza el detector de Kazemi \& Sullivan que mediante un conjunto de árboles de regresión permite encontrar la alineación del rostro (librería dlib en python). La característica que se detecta es el inicio y fin de las cejas. Se procede a estimar el área de análisis, la cual corresponde a un área promedio de 800 pixeles cuadrados, cuando la persona se encuentra a una distancia estimada de $50 \mathrm{~cm}$ de la cámara. Al iniciar la medición y bajo la premisa que el sujeto está a $50 \mathrm{~cm}$ de la cámara se toma las siguientes distancias: primero entre los inicios de las cejas, medida $a$ (parte superior a la nariz) y segundo los finales de las mismas, medida $b$. Estas distancias van a ser nuestro referente ya que es una característica diferente para cada sujeto. Luego mediante la aplicación del modelo Pin-Hole se ajusta el tamaño del área en relación de la distancia del sujeto a la cámara. Si el sujeto se aleja de la cámara la ROI crecerá, en el caso contrario si se acerca decrecerá en busca de analizar siempre la misma región de piel en el sujeto.

Ahora en los caso de cabeceo, guiñada o alabeo de la cabeza del sujeto, también se mantiene la misma región de la piel para analizar el área de la ROI, siempre que la posición del rostro lo permita. En el caso del cabeceo la distancia entre los puntos medios de las medidas $a$ y $b$ se denominará medida $c$, ahora la componente $c y$, se reducirá o ampliara, con lo que la dimensión de la ROI se ensanchara y la distancia entre la medida $a$ y la ROI variara proporcionalmente (la ROI esta paralela a la medida $a$ y la distancia entre estas dos son las que variarán). Aunque no se mantenga la misma región de la piel, tampoco se pierde área de análisis o se introduce ruido (pelo dentro de la ROI). En el caso de la guiñada, la ROI se moverá con el rostro. $\mathrm{Y}$ finalmente para el caso del alabeo o balanceo con la información del ángulo de la dimensión $b$, se procede a calcular la inclinación de la cabeza, alineando el área de análisis a la inclinación del rostro. Así se logra un análisis fotopletismográfico continuo, aproximadamente con el mismo tamaño de área y de la misma región en la frente del sujeto.

Ahora, de la ROI se separan los canales RGB, se realiza el promedio de todos los pixeles. Con este promedio se generan pilas de tipo FIFO, con una dimensión de 6 s. Es un valor seleccionado por ser el tiempo suficiente para contar con un mínimo de 6 pulsos cardiacos. Una vez llena la pila se procede a calcular el offset de la señal mediante el método de Kalman. La aplicación de este filtro es excelente por tres razones: primero la señal promediada están cambiando continuamente. Segundo, a partir de las medidas pasadas y la medida presente, se estima el estado del sistema y no es necesario mantener ningún tipo de historia que no sea el estado anterior. Y tercero, es un algoritmo ligero, lo cual lo hace rápido y aplicable en sistemas de funcionamiento en línea. Se puede observar en la Figura 3, tanto la señal fotopletismográfica promediada y la señal de seguimiento mediante el filtro de Kalman.

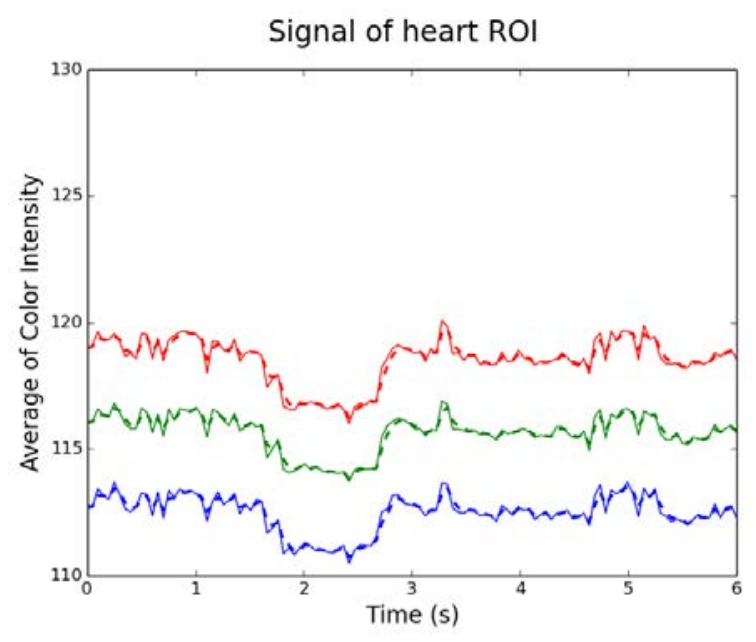

Figura 3: Señal promedio (línea continua) y Señal de seguimiento propuesta (línea entrecortada), en los canales RGB.

Se genera así un filtro de frecuencias bajas, al restar la señal fotopletismográfica y la señal de seguimiento por Kalman. Que es estable, con un tiempo inicial de estabilización rápido, sensible a cambios de la señal y con un coste computacional bajo.

Después de obtener la señal, un filtro de banda estrecha atenúa las frecuencias fuera de la banda de interés. En nuestro caso las frecuencias de corte se presentan en la Tabla 2, son los rangos normales de la frecuencia cardíaca. Los filtros de banda estrecha son de tipo IIR. Butterworth es elegido por los anchos de banda que requieren un orden inferior. Esto significa que hay menos cálculos, lo que conlleva a reducir el coste computacional. 
Se continúa a la vez actualizando las pilas, con el nuevo promedio de cada nueva imagen. Con la nueva señal filtrada se realiza la Transformada Rápida de Fourier FFT. La dimensión de la pila no es potencia de dos, se procede a realizar un relleno de ceros (zero padding), con lo cual se acelera el cálculo de la FFT y además se mejora la resolución de la misma. Se procede a buscar el pico de frecuencia en los tres canales, si su potencia es superior a la deseada se toma como datos válidos.

Si los tres canales contienen el mismo pico de frecuencia o similar, la medida es inequívoca, en el caso de que no se correspondan el nivel de confianza de la medida es menor, pero se toma la del canal $G$ por contener la mayor información, por la configuración del filtro Bayer de la cámara.

Tabla 2: Detalle de población del experimento.

\begin{tabular}{|c|c|}
\hline & Frecuencia \\
\hline Niños & $1,00 \mathrm{~Hz}$ a $2,33 \mathrm{~Hz}$ \\
\hline Adultos & $0,83 \mathrm{~Hz}$ a $1,67 \mathrm{~Hz}$ \\
\hline
\end{tabular}

Finalmente, se realiza la corrección de la medición, mediante la minimización de error con un filtrado con Kalman que se encarga de eliminar los picos de frecuencia indeseados cuando el nivel de confianza es bajo, para así visualizar la medida, Figura 4.

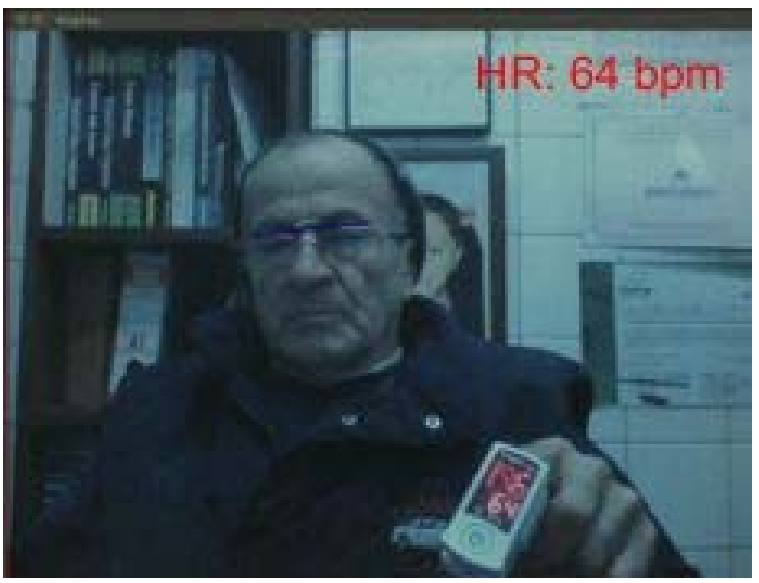

Figura 4: Visualización del sistema en funcionamiento.

\section{RESULTADOS}

Con el fin de comprobar el comportamiento del sistema, se realizaron las mediciones con nuestra metodología y se efectúa la siguiente evaluación. Mediciones en línea de los individuos descritos en la Tabla 1, por nuestro método y por el pulsímetro de dedo. Se toman mediciones cada segundo durante un periodo de tiempo mayor a 60 segundos. Se utiliza el método de Bland y Altman que permite comparar las mediciones realizadas tanto por el pulsímetro de dedo, así como por nuestro método. Por la cantidad de muestras se utiliza el método con el análisis de los ratios y las medias de las dos técnicas. Los resultados se representan en la Figura 5 y Tabla 3.

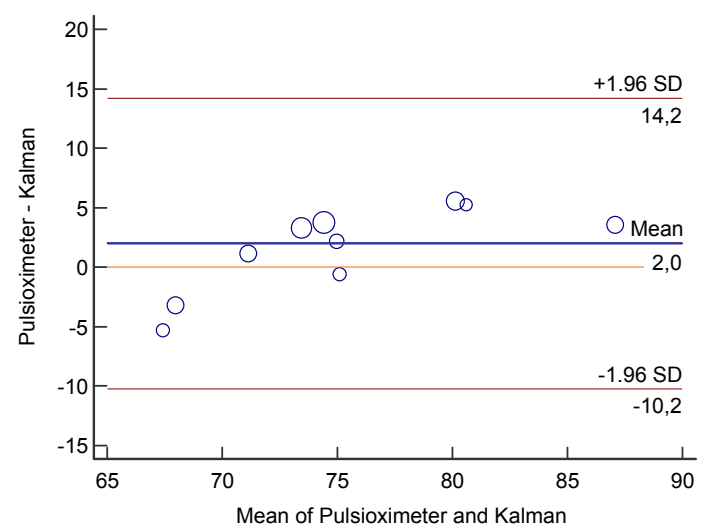

Figura 5: Evaluación mediante Bland y Altman entre el pulsímetro de dedo y el ritmo cardíaco calculado con nuestro método.

Tabla 3: Datos estadísticos de la evaluación.

\begin{tabular}{|l|r|}
\hline & Valores \\
\hline Tamaño de Muestra & 1887 \\
\hline Media Aritmética & 1,9995 \\
\hline Desviación Estándar & 6,2297 \\
\hline Límite Inferior & $-10,2107$ \\
\hline Límite Superior & 14,2097 \\
\hline
\end{tabular}

\section{DISCUSIÓN}

En resumen, los resultados presentados aquí indican que la medida de la frecuencia cardíaca mediante el análisis PPGI con el método de Kalman como filtro de frecuencias bajas es un método robusto y con un coste computacional bajo. Por lo cual se ha presentado la comparación y evaluación de varias mediciones realizadas en línea. Es un punto fundamental de nuestro sistema en comparación con otros sistemas similares por PPGI que tienen un coste computacional elevado, por lo cual casi no existen sistemas que funcionen en línea. Además, son susceptibles a las variaciones de luz y el movimiento. Todo esto ha sido superado en gran medida en el método aquí presentado, falta aún mucho camino por recorrer pero este método puede ser la base de futuras mejoras, para conseguir un sistema de medición del ritmo cardíaco completamente fiable por PPGI.

Lo fundamental en la imagen fotopletismográfica es que al no tener relación con los métodos de medición clásicos se evita producir molestias o incomodidades a los pacientes, pues es una medición imperceptible y sin contacto para los mismos. Y se cuenta con la ventaja que hay en la señal fotopletismográfica la 
información necesaria para encontrar la saturación de oxígeno y presión sanguínea, línea de investigación en la cual se está trabajando actualmente.

Los resultados de este experimento han demostrado la robustez de nuestro algoritmo. Además, nuestra metodología difiere en su evaluación ya que se observa claramente que otros métodos propuestos se evaluaron dentro de ambientes controlados en los cuales no existen variaciones de luz, sombras o movimientos naturales por parte de los sujetos grabados, ya que se encuentran conectados a diversos equipos de medición. Por lo que, en este trabajo se realizó el análisis de 10 individuos, en situaciones más reales como: distintos tipos de iluminación (natural, incandescente, fluorescente), aparición de sombras, grabaciones contra luz, variaciones de intensidad lumínica.

Además, existe una interacción humana con los sujetos, por lo cual se observa en ellos gestos, risas, movimientos más naturales entre otros. Se analizan estos datos recogidos, se validan con la obtención de información sobre el rendimiento y la fiabilidad del sistema. Todo esto mediante el método de Bland y Altman, Figura 5. Hay una ligera diferencia sistemática entre las dos mediciones. Por un lado, debido al equipo de medición estándar, un pulsímetro de dedo con una precisión de \pm 3 puntos porcentuales, según especificaciones del fabricante. También, algunos valores atípicos, causadas por fuertes movimientos de los sujetos, la no detección del rostro en algunos momentos y por la tonalidad de la piel de otros que dificulta la medida. No se debe olvidar que la mayor concentración de melanina hace imperceptible las variaciones de la hemoglobina, ya que su coeficiente de absorción es mayor.

\section{CONCLUSIONES}

Como ya se mencionó anteriormente, el presente sistema de medición del ritmo cardíaco puede convertirse en un estándar o base para los sistemas de imagen fotopletismografía, por su coste computacional bajo, su gran robustez y eficacia. Se espera evaluarlo posteriormente comparándolo con otras metodologías por PPGI y en situaciones más reales, como la medición de la frecuencia cardíaca del paciente en movimiento junto con la cámara web y a la vez cambiando de ambientes e iluminación.

Se trabaja actualmente en la medición de la saturación del oxígeno en línea y otros parámetros fisiológicos. Se espera en el futuro, seguir mejorando las mediciones, mediante el análisis en paralelo de diversas regiones de la piel y un sistema de valoración y comparación de dichas señales.

\section{Agradecimientos}

El trabajo ha sido financiado parcialmente por el proyecto RoboCity2030-III-CM (Robótica aplicada a la mejora de la calidad de vida de los ciudadanos. fase III; S2013/MIT-2748) financiado por Programas de Actividades $\mathrm{I}+\mathrm{D}$ en la Comunidad de Madrid y cofinanciado por los Fondos Estructurales de la Unión Europea.

Además, el primer autor agradece al Gobierno Ecuatoriano, por la beca que apoya y financia sus estudios. Su gratitud también a la ayuda directa de los laboratorios de la Universidad Carlos III de Madrid.

\section{Referencias}

[1] M. Adibuzzaman, S. I. Ahamed and R. Love. Proceedings of the 29th annual ACM symposium on applied computing - SAC '14'; A personalized model for monitoring vital signs using camera of the smart phone. 2014. DOI: $10.1145 / 2554850.2555019$

[2] Duc Nhan Tran, Hyukzae Lee and Changick Kim. 2015 IEEE international conference on multimedia and expo (ICME); A robust real time system for remote heart rate measurement via camera. 2015. DOI: 10.1109/ICME.2015.7177484.

[3] A. Lam and Y. Kuno. Robust heart rate measurement from video using select random patches. Presented at Proceedings of the IEEE International Conference on Computer Vision. 2015.

[4] M. Lewandowska, J. Rumiński and T. Kocejko. Measuring pulse rate with a webcam-a noncontact method for evaluating cardiac activity. Presented at Computer Science and Information Systems (FedCSIS), 2011 Federated Conference On. 2011.

[5] X. Li, J. Chen, G. Zhao and M. Pietikainen. Remote heart rate measurement from face videos under realistic situations. Presented at Proceedings of the IEEE Conference on Computer Vision and Pattern Recognition. 2014.

[6] M. Z. Poh, D. J. McDuff and R. W. Picard. Advancements in noncontact, multiparameter physiological measurements using a webcam. IEEE Trans. Biomed. Eng. 58(1), pp. 7-11. 2011. DOI: 10.1109/TBME.2010.2086456 [doi]. 
[7] M. Z. Poh, D. J. McDuff and R. W. Picard. Non-contact, automated cardiac pulse measurements using video imaging and blind source separation. Opt. Express 18(10), pp. 10762-10774. 2010. DOI: 10.1364/OE.18.010762 [doi].

[8] W. Verkruysse, L. O. Svaasand and J. S. Nelson. Remote plethysmographic imaging using ambient light. Opt. Express 16(26), pp. 21434-21445. 2008. DOI: 175396 [pii].

[9] H. Wu, M. Rubinstein, E. Shih, J. V. Guttag, F. Durand and W. T. Freeman. Eulerian video magnification for revealing subtle changes in the world. ACM Trans.Graph. 31(4), pp. 65. 2012.

[10] S. Xu, L. Sun and G. K. Rohde. Robust efficient estimation of heart rate pulse from video. Biomed. Opt. Express 5(4), pp. 1124-1135. 2014. DOI: 10.1364/BOE.5.001124 [doi]. 